\title{
Pax6-5a Promotes Neuronal Differentiation of Murine Embryonic Stem Cells
}

\author{
Nao Shimizu, ${ }^{a, b}$ Hajime Watanabe ${ }^{c}$ Junko Kubota, ${ }^{b}$ Jinzhan Wu,${ }^{a, b}$ Ryota Saito, ${ }^{a, b}$ Tadashi Yokoi, ${ }^{a, g}$ \\ Takumi Era, ${ }^{d}$ Takeshi Iwatsubo, ${ }^{e}$ Takashi Watanabe ${ }^{f}$ Sachiko Nishina, ${ }^{g}$ Noriyuki Azuma, ${ }^{*, g}$ \\ Toshiaki KaTADA, ${ }^{b}$ and Hiroshi NiSHINA ${ }^{*, a}$ \\ ${ }^{a}$ Department of Developmental and Regenerative Biology, Medical Research Institute, Tokyo Medical and Dental \\ University; Tokyo 113-8510, Japan: ${ }^{b}$ Department of Physiological Chemistry, Graduate School of Pharmaceutical \\ Sciences, University of Tokyo; ${ }^{e}$ Department of Neuropathology and Neuroscience, Graduate School of Pharmaceutical \\ Sciences, University of Tokyo; Tokyo 113-0033, Japan: ${ }^{c}$ Center for Integrative Bioscience, Okazaki National Research \\ Institutes; Okazaki 444-8787, Japan: ' Department of Organogenesis, Institute of Molecular Embryology and Genetics, \\ Kumamoto University; Kumamoto 860-0811, Japan: ${ }^{f}$ Department of Laboratory Medicine, Kyorin University School of \\ Medicine; Tokyo 181-8611, Japan: and ${ }^{g}$ Department of Ophthalmology, National Center for Child Health and \\ Development; Tokyo 157-8535, Japan. Received January 16, 2009; accepted March 11, 2009
}

Pax6 genes are highly conserved and important for eye development. Vertebrates predominantly produce two alternatively spliced Pax6 isoforms, Pax6 and Pax6-5a. Pax6-5a differs from Pax6 by the presence of 14 additional amino acids encoded by exon 5a. These additional amino acids occur in the Pax6 paired domain and thus influence its DNA-binding properties. However, little is known about Pax6-5a's physiological functions. Here we establish murine embryonic stem (ES) cell lines in which expression of either the human Pax6 or Pax6-5a isoform is negatively controlled by tetracycline. We report that, in contrast to Pax6 expression, Pax6-5a expression strongly induces ES cells to differentiate into neurons. Moreover, using DNA microarray analysis, we have identified the transcription factor basic helix loop-helix domain containing, class b2 (bHLHb2) in Pax6-5a-expressing ES cells. Our Pax6 isoform-expressing ES cell lines may serve as useful models for identifying Pax6-regulated genes that are important for neurogenesis and/or eye development.

Key words Pax6; Pax6-5a; embryonic stem cell; neuron; DNA microarray; basic helix loop-helix domain containing, class b2

Pax6 is a transcription factor essential for the development of the eye, brain and pancreas. ${ }^{1,2)}$ Pax6 is defined by the presence of its paired domain, a highly conserved DNA-binding motif composed of two distinct DNA-binding subdomains called the N-terminal subdomain (NTS) and the C-terminal subdomain (CTS). The NTS and the CTS bind to distinct consensus DNA sequences. ${ }^{3,4)}$ Transcription of the human Pax6 gene results in two alternatively spliced isoforms, Pax6 and Pax6-5a. Compared to Pax6 transcripts, Pax6-5a transcripts contain an additional exon 5 a that encodes an additional 14 amino acids. These amino acids are inserted into the NTS, an event that abolishes the DNA-binding activity of this subdomain and unmasks the DNA-binding activity of the CTS. Thus, exon 5a transcription appears to function as a molecular switch that regulates the spectrum of Pax6 target genes that can be induced. Previously, we reported a missense mutation in the exon 5a region of the Pax6 gene in patients with Peters anomaly. This mutation resulted in a Pax6 protein with impaired DNA-binding and transactivation activities. 5) Subsequently, we found that although overexpression of either Pax6 or Pax6-5a in chick embryos induced transdifferentiation of ectopic neural retina from primitive retinal pigment epithelium, Pax6-5a was a much stronger driver of this process than was Pax6. ${ }^{6,7)}$ However, little is known about how Pax6 and Pax6-5a actually function in vivo.

Embryonic stem (ES) cells are pluripotent cells derived from the inner cell mass of preimplantation mouse embryos. ES cells can be propagated stably in the undifferentiated state in vitro and, under the appropriate culture conditions, can be induced to differentiate into a variety of cell types. For example, forced transgenic expression of Pdx1 causes ES cells to differentiate into pancreatic cells (endoderm), whereas GATA2 expression promotes leukocyte differentiation (mesoderm), and Mash1 expression induces neuronal differentiation (ectoderm). ${ }^{8,9)}$ This plasticity makes ES cell culture a useful tool for elucidating the functions of transcription factors.

In this report, we have employed conditional expression of human Pax6-5a driven by a tetracycline-regulatable promoter and have found that forced Pax6-5a expression in murine ES cells enhances their differentiation into neuronal cells. Our system may be useful for uncovering the molecular mechanisms underlying Pax6-5a-dependent eye development.

\section{MATERIALS AND METHODS}

Establishment of Tet-Regulated ES Cell Clones The murine ES cell line E14tg2a was maintained in Dulbecco's modified Eagle's medium (DMEM) supplemented with 15\% fetal calf serum, $0.1 \% \quad \beta$-mercaptoethanol, and $1000 \mathrm{U} / \mathrm{ml}$ leukemia inhibitory factor (LIF). The tetracycline (Tet) regulatory system was used to obtain inducible Pax6- or Pax6-5aexpressing ES cell clones as described previously. ${ }^{10)}$ Briefly, Tet-regulatable Flag-Pax6 and Flag-Pax6-5a constructs were generated by inserting Flag-human Pax6 or Flag-human Pax6-5a cDNA into the NotI site of pUHD10-3.IRES-EGFP. Expression of these Tet-regulatable constructs in ES cells was completely suppressed by the addition of tetracycline to the culture medium. The ES cell clones whose enhanced green fluorescent protein (EGFP) expression was most tightly regulated by tetracycline were selected for further study, including examination of the expression of relevant genes by reverse transcription-polymerase chain reaction 
(RT-PCR) and Western blotting.

ES Cell Differentiation Embryoid bodies (EBs) were prepared as described previously. ${ }^{11)}$ Briefly, undifferentiated ES cells were dissociated into single-cell suspensions and cultured in hanging drops to induce embryoid body (EB) formation. Initial cell density was 2000 cells per drop $(25 \mu \mathrm{l})$ of differentiation medium without LIF in the absence or presence of $1 \mu \mathrm{g} / \mathrm{ml}$ Tet (Day 0 ). After 2 days in a hanging drop culture (Day 2), the resulting EBs were transferred to noncoated culture dishes. On Day 3, bFGF (20 ng/ml, R\&D Systems) was added to the culture medium. On Day 7, the EBs were plated in plastic gelatin-coated dishes.

Immunoprecipitation and Immunoblotting For detection of induced Flag-Pax6 and Flag-Pax6-5a proteins, ES cells were cultured for $48 \mathrm{~h}$ in the absence of Tet. ES cells $\left(5 \times 10^{6}\right)$ were then lysed and immunoprecipitated with antiFlag M2 antibody (Ab) (Sigma, F3165). The immunoprecipitates were fractionated by sodium dodecyl sulfate-polyacrylamide gel electrophoresis (SDS-PAGE) and immunoblotted with anti-Flag M2 Ab. Bands were visualized using the SuperSignal West Pico chemiluminescent substrate according to the manufacturer's instructions (PIERCE, IL, U.S.A.).

Confocal Microscopy Immunofluorescence studies were performed as described previously. ${ }^{12}$ Briefly, EBs cultured on gelatin-coated dishes were washed three times with phosphate buffered saline (PBS) and fixed in 4\% paraformaldehyde. After permeabilization with $0.2 \%$ Triton X-100, the EBs were incubated with blocking solution [5\% bovine serum albumin (BSA) in TBS], followed by anti- $\beta$ III-tubulin $\mathrm{Ab}$ (Covance, MMS-435P). The stained EBs were washed with PBS and incubated with Alexa-568-conjugated secondary $\mathrm{Ab}$ (Molecular Probes). After a last wash in PBS, EBs were viewed on a Carl Zeiss confocal microscope equipped with LSM510 software.

RT-PCR Analysis ES cells were lysed with Trizol reagent (Invitrogen) and first-strand cDNA was synthesized by using SuperScript 111 RNase H-reverse transcriptase (Invitrogen). Primers used were: Flag-Pax6, 5'-ATGGATTACAAGGATGACGACG-3' and 5'-ATCTGTTGCTTTTCGCTAGCC-3'; Acetylcholine esterase (Ache), 5'-CCGATTTTCCTTCGTGCCTG-3' and 5'-TGGAGGCACGGTGTTCAAAG-3'; Serotonin receptor 5a (5-HT5a), 5'-AGTCGGCCTTTTCTCTCAGC-3' and 5'-GGTCCAGTGCTATTGCTGTC-3'; L1, 5'-AGGACACCATGTGCTAGAGC-3' and 5'GGGTTGCAAGGCAGAACTAC-3'; Elongation factor 1 (EF1), 5'-TCACACAGCCCACATAGCAT-3' and 5'-CACCACTGATTAAGACTGGG-3'; Oct3/4, 5'-CTGAGGGCCAGGCAGGAGCACGAG-3' and 5'-CTGTAGGGAGGGCTTCGGGCACTT-3'; bHLHb2, 5'-TTATTGCACAGCTAGACACGG-3' and 5'-ACACTGTAACCTCGCCTCTC-3'.

DNA Microarray Total RNAs from EBs were extracted using TRIZOL (Invitrogen, Tokyo, Japan) and purified using an RNeasy mini kit (Qiagen, Tokyo, Japan). Purified RNA was labeled with biotin according to the manufacturer's protocol and hybridized to a mouse genome 430A 2.0 array (Affymetrix Japan, Tokyo, Japan). After washing, the array was scanned to measure fluorescence intensity (representing gene expression). The fluorescence intensity of each probe was further analyzed by dChip, a model-based expression analysis program that estimates gene expression levels. For the dChip analysis, a perfect match (PM)-only model was used. The estimated gene expression level values were applied to the Gene-Spring software program (Silicon Genetics, Redwood City, CA, U.S.A.). ${ }^{13)}$

Construction of Plasmids A DNA fragment encompassing -90 to $-1996 \mathrm{bp}$ of the $5^{\prime}$ region of the murine bHLHb2 gene was subjected to PCR amplification using the iProof High Fidelity DNA Polymerase system (BioRad). After digestion with $M l u \mathrm{I}$ and $X h o \mathrm{I}$, the PCR product was subcloned into the $M l u \mathrm{I} / X h o \mathrm{I}$ sites of the pTA-Luc vector (CLONTECH), resulting in pbHLHb2 (-90_-1996). To construct the pTA-5aCON-Luc vector, which served as a positive control for the Pax6-5a reporter assay, the oligonucleotides 5'-AAATCTGAACATGCTCAGTGAATGTTCATTGACTCTCGAGGTC-3' and 5'-GACCTCGAGAGTCAATGAACATTCACTGAGCATGTTCAGATTT-3' were annealed, phosphorylated and ligated into the $S m a$ I site of the pTA-Luc vector. Underlining indicates Pax6-5a binding sequences.

Transient DNA Transfections and Luciferase Reporter Assays DNA transfections were carried out using the Lipofectamine 2000 reagent (Invitrogen). Briefly, the human embryonic carcinoma cell line NTERA2 were plated in a 24-well plate. After $24 \mathrm{~h}$ transfection with the above reporter plasmids, the cells were harvested and firefly and sea pansy luciferase activities were determined using the dualluciferase reporter assay system (Promega) and a Wallac ARVO.SX 1420 Multilabel Counter (Amersham Pharmacia Biotech). All experiments were repeated at least three times with different batches of transfected cells and the results were fully reproducible.

\section{RESULTS}

Tet-regulatable Pax6 and Pax6-5a Expression To investigate the biological functions of Pax6 and Pax6-5a, we established murine ES cell lines with tetracycline (Tet)-regulatable Flag-human Pax6 or Flag-human Pax6-5a expression. To this end, a Tet-regulatable bicistronic vector encoding Flag-Pax6 or Flag-Pax6-5a plus the EGFP was introduced into ES cells. ES cell lines were then established in the presence of Tet (Tet-on) to suppress the expression of the exogenous Flag-Pax6 or Flag-Pax6-5a. Conditional expression of Flag-Pax6 or Flag-Pax6-5a and EGFP in these ES cells was achieved by withdrawal of Tet (Tet-off). The successful expression of Flag-Pax6 and Flag-Pax6-5a proteins in Tet-off ES cell lines was confirmed by Western blot analysis using anti-Flag antibody (Fig. 1A), whereas EGFP expression was detected with confocal microscopy (Fig. 1B). These results confirm that our Pax6 isoform-expressing ES cell lines showed EGFP plus exogenous Pax6 or Pax6-5a protein expression that was fully regulated by Tet.

Pax6-5a Expression Enhances Neuronal Differentiation by ES Cells Cultured murine ES cells can be induced to differentiate in vitro into a variety of cell types, including cardiac cells and neurons. To separately examine the effects of Pax6 and Pax6-5a on ES cell differentiation, we cultured our Tet-regulatable ES cell lines without LIF in the presence or absence of Tet and allowed them to form embryoid bodies (EBs). Interestingly, Tet-regulatable Pax6-5a-expressing ES cells differentiated into neuron-like cells in absence, but not presence, of Tet (Fig. 2A). To confirm that the observed dif- 


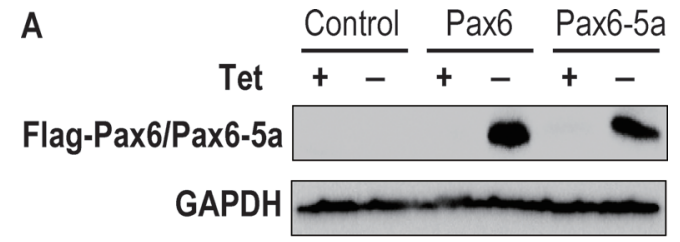

B

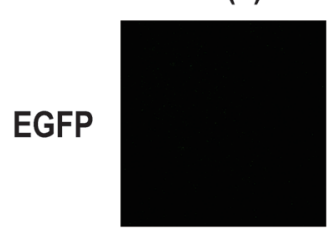
Tet $(-)$

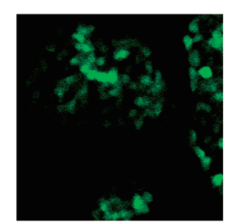

Phase
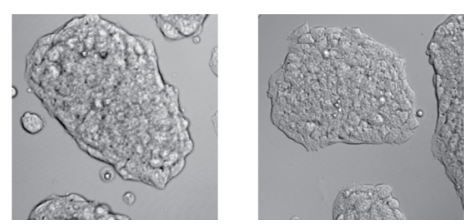

Fig. 1. Establishment of Murine ES Cell Lines with Tet-Regulatable Expression of Pax6 Isoforms

(A) Confirmation of Tet-regulated expression by Western blot. Murine ES cell lines expressing either EGFP vector alone (control) or EGFP plus either Flag-human Pax6 (Pax6) or Flag-human Pax6-5a (Pax6-5a) were cultured with (+) or without (-) Tet for $2 \mathrm{~d}$ and subjected to Western blotting using anti-Flag Ab. GADPH, loading control. (B) Confirmation of transfection. Detection of EGFP expression in monolayers of Tet-regulatable Pax6-5a-expressing ES cells in the presence or absence of Tet. Top, confocal microscopy; bottom, phase contrast microscopy. For all figures, results shown are one trial representative of at least three independent experiments.

ferentiating cells were in fact neurons, we immunostained them with anti- $\beta$ III-tubulin $\mathrm{Ab}$ and obtained positive results (Fig. 2A, right panel). On the other hand, this type of neuronal differentiation did not occur in Tet-off Pax6-expressing ES cells (data not shown). We then used RT-PCR to examine the expression by both of our Tet-regulatable ES cell lines of typical neuronal marker genes such as acetylcholinesterase (Ache; cholinergic neurons), serotonin receptor 5a (5-HT5a; serotonergic neurons), and L1 (neuronal cell adhesion molecule). Expression levels of Ache, 5-HT5a and L1 mRNAs were increased in Tet-off Pax6-5a-expressing ES cells, but not in Tet-on Pax6-5a-expressing ES cells, nor in Tet-off or Tet-on Pax6-expressing ES cells (Fig. 2B). These data indicate that expression of Pax6-5a by murine ES cells enhances their neuronal differentiation.

Pax6-5a Expression Enhances Neurite Development by Murine EBs To determine the timing of Pax6-5a-induced neuronal commitment, we examined neurite development in EB cultures of Pax6-5a-expressing ES cells from which Tet was removed for $0,1,2,3,4$ or $11 \mathrm{~d}$ after Day 0 (Fig. 3A). When Tet was withdrawn for $11 \mathrm{~d}$, more than $60 \%$ of EBs were found to be neurite-positive (Fig. 3B, row f). The most dramatic increase in the percentage of neurite-positive EBs observed occurred between $2 \mathrm{~d}$ (condition $\mathrm{c}$ ) and $3 \mathrm{~d}$ (condition d) of Tet withdrawal, suggesting that Pax6-5a-induced neuronal commitment is established in this system during the first $3 \mathrm{~d}$ of culture.

Pax6-5a Expression Upregulates Neuron-Related Genes in Murine EBs To determine the identity of genes activated downstream of Pax6-5a, we performed a DNA mi-

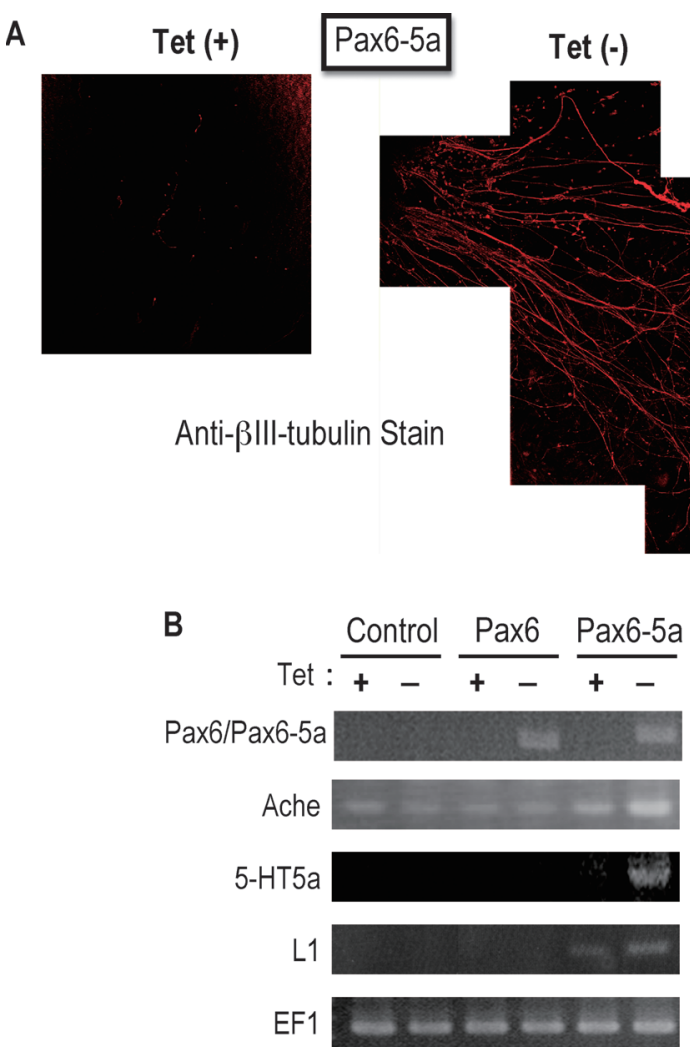

Fig. 2. Pax6-5a Enhances the Neuronal Differentiation of Murine ES Cells

(A) Neuron-like differentiation. Tet-regulatable Pax6-5a-expressing ES cells were allowed to form EBs in the presence or absence of Tet, followed by immunostaining with anti- $\beta$ III-tubulin Ab. (B) Neuronal marker expression. Control, Pax6- and Pax6-5a-expressing ES cell lines were cultured in the presence or absence of Tet for $11 \mathrm{~d}$ and expression levels of the neuronal marker genes Ache, 5-HT5a and L1 were determined by RT-PCR. EF1, loading control.

A
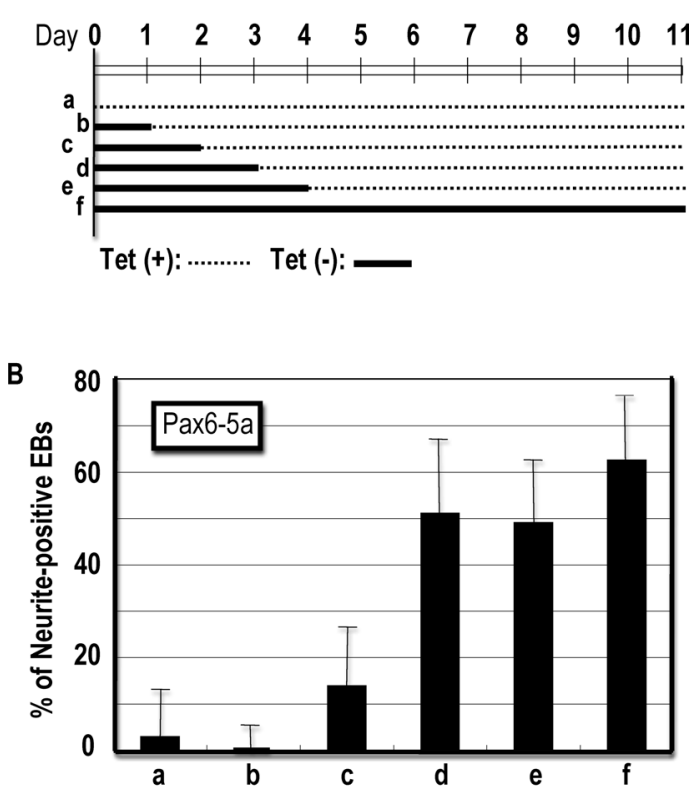

Fig. 3. Time Course of Pax6-5a-Induced Commitment to ES Cell Neuronal Differentiation

(A) Experimental scheme. Tet-regulatable Pax6-5a-expressing ES cells were cultured with Tet for $0-11 \mathrm{~d}$ as indicated $(\mathrm{a}-\mathrm{f})$. EB formation commenced on Day 0 and neuronal differentiation was assessed on Day 11. (B) Day 3 commitment. Percentages of neurite-positive EBs in the cultures in (A) were assessed by microscopy. A significant increase in neurite-positive EBs occurs between Day 2 and Day 3. 
croarray analysis of Tet-regulatable Pax6-5a ES cells cultured for $3 \mathrm{~d}$ in the absence or presence of Tet. Among the many genes whose expression was increased in a Pax6-5adependent manner were the transcription factors basic helixloop-helix domain containing, class b2 (bHLHb2) and Oct3/4 (data not shown). Both bHLHb2 and Oct3/4 are able to induce ES cells to differentiate into neuronal cells. ${ }^{14,15)}$ To confirm the effects of Pax6-5a on the expression of bHLHb2 and Oct3/4, we carried out an RT-PCR time course analysis of Tet-regulatable Pax6- or Pax6-5a-expressing ES cells cultured in the presence or absence of Tet for $1-5 \mathrm{~d}$. bHLHb2 mRNA expression was constant in Tet-on and Tet-off Pax6expressing ES cells regardless of the number of days of Tet withdrawal, whereas Oct3/4 expression declined in a timedependent manner in both Tet-on and Tet-off Pax6-expressing ES cells (Fig. 4A, upper panel). In contrast, expression levels of both bHLHb2 and Oct3/4 mRNAs were increased in Tet-off Pax6-5a-expressing ES cells compared to Tet-on Pax6-5a-expressing ES cells (Fig. 4A, lower panel). These results suggest that $b H L H b 2$ and $O c t 3 / 4$ are downstream target genes of Pax6-5a.

To determine whether the $b H L H b 2$ gene was truly a transcriptional target of Pax6-5a, we carried out luciferase reporter assays examining $b H L H b 2$ promoter activity. A DNA
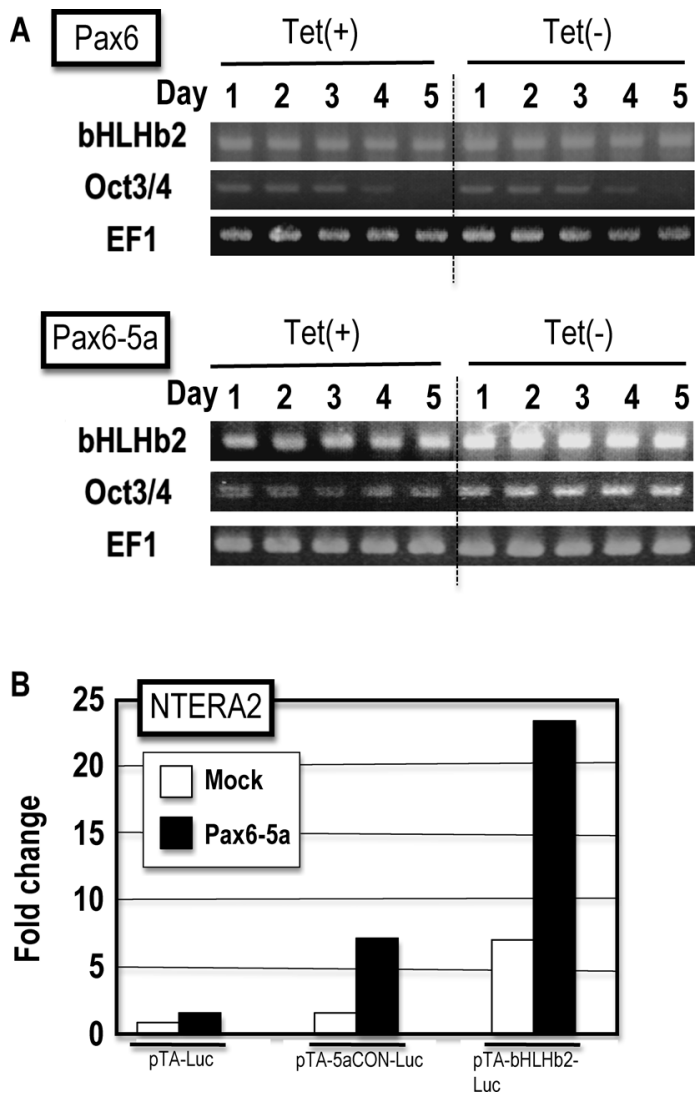

Fig. 4. Influence of Pax6-5a on bHLHb2 and Oct3/4 Gene Expression and bHLHb2 Transcriptional Activity

(A) Increased $b H L H b 2$ and Oct3/4 expression. Tet-regulatable Pax6- or Pax6-5a-expressing ES cells were cultured for the indicated times in the presence or absence of Tet and levels of bHLHb2 and Oct3/4 mRNAs were assessed by RT-PCR. (B) $b H L H b 2$ is a Pax6-5a target gene. NTERA2 cells were co-transfected with a plasmid expressing Pax6-5a or the vehicle alone (mock), plus the indicated luciferase reporter constructs: pTA-Luc (negative control), pTA-5aCON-Luc (positive control) or pTA-bHLHb2-Luc ( $5^{\prime}$ region of the $b H L H b 2$ gene). Results shown are the fold change in luciferase activity relative to negative control. fragment encompassing -90 to $-1996 \mathrm{bp}$ of the $5^{\prime}$ region of the murine $b H L H b 2$ gene was positioned upstream of the pTA-Luc firefly luciferase reporter gene to create the pTAbHLHb2-Luc reporter. This reporter was cotransfected along with a plasmid expressing Pax6-5a into NTERA2 cells, a human embryonic carcinoma cell line. As a positive control, we generated the pTA-5aCON-Luc reporter plasmid that contains a Pax6-5a-binding sequence and responds to Pax65a. Interestingly, the degree of induction of pTA-bHLHb2Luc was equivalent to that of pTA-5aCON-Luc, indicating that the $b H L H b 2$ gene is directly or indirectly regulated by Pax6-5a at least in NTERA2 cells.

\section{DISCUSSION}

In this study, we have shed light on the function of the Pax6-5a isoform using a Tet-regulated conditional expression system in the context of EB-mediated ES cell differentiation into neuronal cells. Firstly, we established ES cell lines in which the expression of human Pax6 or Pax6-5a was regulated by the presence or absence of Tet. Secondly, we demonstrated that expression of Pax6-5a, but not Pax6, enhanced the differentiation of EBs, but not monolayer ES cells, into Ache $^{+}$5-HT5a ${ }^{+} \mathrm{L1}^{+}$neuron-like cells. Thirdly, we showed that Pax6-5a-induced neuronal commitment occurs within the first $3 \mathrm{~d}$ of EB formation. Finally, we provisionally identified $b H L H b 2$ and Oct3/4 as downstream target genes of Pax6-5a.

We have previously described a missense mutation within Pax6 exon 5a in four families with members suffering from Peters anomaly, congenital cataracts, Axenfeldt anomaly, and/or foveal hypoplasia. ${ }^{5)}$ Biochemical analysis showed that this mutation caused a decrease in CTS transactivation activity. Singh et al. have reported iris hypoplasia and defects in the cornea, lens and retina in mice lacking Pax6 exon 5a. ${ }^{16}$ On the other hand, overexpression of Pax6-5a induces a remarkably well-differentiated retina-like structure in chick embryos. ${ }^{6)}$ The collective results of these "loss of function" and "gain of function" studies suggest that the evolution of the Pax6-5a isoform has contributed to the advanced features of the vertebrate eye. Our data are consistent with this hypothesis, in that forced expression of Pax6-5a, but not Pax6, strongly induced the neuronal differentiation of ES cells.

As shown in Fig. 1, our established ES cell lines showed EGFP plus exogenous Pax6 or Pax6-5a protein expression that was strictly regulated by Tet in monolayer-culture conditions. However, the percentage of neuronal differentiation from EB culture, which is a kind of three-dimensional culture, were $23 \%$ in the presence of Tet and $97 \%$ in the absence of Tet, respectively (data not shown). We could observe the expression of neuronal marker genes such as L1 and Ache even in the presence of Tet by RT-PCR (Fig. 2B). We speculate that Tet is less effective for EB culture compared to monolayer culture, resulting in a leak of Pax6-5a expression and an induction of neuronal differentiation from EB culture.

As mentioned above, the paired domain of Pax6 is composed of two subdomains: NTS (also called PAI) and CTS (also called RED). Each subdomain recognizes a distinct half-site of the bipartite Pax6 binding site positioned in adjacent major grooves of the DNA. In Pax6-5a, the NTS domain is modified by the insertion of 14 amino acids encoded by 
the additional exon 5a. This addition alters the DNA-binding specificity of the paired domain such that it recognizes a new consensus sequence, $5 \mathrm{aCON}$. The $5 \mathrm{aCON}$ sequence consists of four interdigitated $5^{\prime}$ half-sites of the bipartite consensus sequence and is thus bound by four Pax6-5a molecules via the intact CTS domain. A previous study of transgenic mice overexpressing human Pax6-5a in the lens showed that the human $\alpha 5$ and $\beta 1$ integrin promoters contain both Pax6 and Pax6-5a binding sites and maybe directly regulated by Pax6 isoforms. ${ }^{17)}$ Another report based on a genome database search and biochemical analysis showed that an enhancer present in the $\gamma \mathrm{E}$ - and $\gamma \mathrm{F}$-crystallin genes is recognized by both Pax6 and Pax6-5a. ${ }^{18)}$ However, there is little physiological data on genes that are regulated by Pax6-5a, either directly or indirectly. Therefore, our Tet-regulatable Pax6 isoform-expressing ES cell lines should be useful for identifying target genes of Pax6 and/or Pax6-5a. Indeed, we found that expression levels of Oct $3 / 4$ and hHLHb2 were strikingly influenced by Pax6-5a.

In vivo, Oct $3 / 4$ plays a critical role in maintaining ES cell pluripotency. In vitro, sustained transgenic Oct3/4 expression in ES cells cultured in serum-free LIF-deficient medium causes accelerated differentiation to neuroectoderm-like cells, whereas suppression of Oct3/4 abolishes neuronal differentiation. ${ }^{15)}$ Thus, Oct3/4 promotes neuroectoderm formation and subsequent ES cell differentiation into neuronal cells. Our data indicate that the activation of Oct3/4 necessary for neuroectoderm formation depends on Pax6-5a activity. The other gene we found to be regulated by Pax6-5a, bHLHb2, encodes a basic helix-loop-helix protein with significant sequence similarities to the Drosophila hairy and enhancer-of-split proteins as well as mouse Hes proteins. Overexpression of bHLHb2 in monolayers of P19 embryonal carcinoma cells results in neuronal differentiation under conditions where P19 cells typically undergo only mesodermal/ endodermal differentiation. ${ }^{14)}$ Thus, bHLHb2 is thought to be a repressor of several of the cell fate decisions that occur during cellular differentiation. Our data indicate that Pax6-5a may regulate bHLHb2 activation and thus determine neuronal differentiation during embryogenesis. With respect to adult vertebrates, Pax6 has been shown to be required for the production and maintenance of progenitor cells during postnatal hippocampal neurogenesis. ${ }^{19)}$ Thus, Pax6 plays important roles in both embryogenesis and adult body homeostasis. Our results suggest that Pax6-5a may be the Pax6 isoform most critical for influencing the neuronal differentiation nec- essary for these processes.

Acknowledgments This work was supported in part by research grants (to H.N.) from the Ministry of Education, Culture, Sports, Science and Technology (MEXT) of Japan, and the Japan Society for the Promotion of Science (JSPS). We are grateful to numerous members of the Nishina and Katada laboratories for critical reading and helpful discussions.

\section{REFERENCES}

1) Gehring W. J., Int. J. Dev. Biol., 46, 65-73 (2002).

2) Pichaud F., Desplan C., Curr. Opin. Genet. Dev., 12, 430-434 (2002).

3) Epstein J. A., Cai J., Glaser T., Jepeal L., Maas R. L., J. Biol. Chem., 269, 8355-8361 (1994).

4) Epstein J. A., Glaser T., Cai J., Jepeal L., Walton D. S., Maas R. L., Genes Dev., 8, 2022-2034 (1994).

5) Azuma N., Yamaguchi Y., Handa H., Hayakawa M., Kanai A., Yamada M., Am. J. Hum. Genet., 65, 656-663 (1999).

6) Azuma N., Tadokoro K., Asaka A., Yamada M., Yamaguchi Y., Handa H., Matsushima S., Watanabe T., Kohsaka S., Kida Y., Shiraishi T., Ogura T., Shimamura K., Nakafuku M., Hum. Mol. Genet., 14, 735745 (2005)

7) Azuma N., Tadokoro K., Asaka A., Yamada M., Yamaguchi Y., Handa H., Matsushima S., Watanabe T., Kida Y., Ogura T., Torii M., Shimamura K., Nakafuku M., Hum. Mol. Genet., 14, 1059-1068 (2005).

8) Kitajima K., Masuhara M., Era T., Enver T., Nakano T., EMBO J., 21, 3060-3069 (2002)

9) Kanda S., Tamada Y., Yoshidome A., Hayashi I., Nishiyama T., Int. J. Dev. Neurosci., 22, 149-156 (2004).

10) Era T., Witte O. N., Proc. Natl. Acad. Sci. U.S.A., 97, 1737-1742 (2000).

11) Takayanagi H., Kim S., Koga T., Nishina H., Isshiki M., Yoshida H., Saiura A., Isobe M., Yokochi T., Inoue J., Wagner E. F., Mak T. W., Kodama T., Taniguchi T., Dev. Cell, 3, 889-901 (2002).

12) Kitagawa D., Kajiho H., Negishi T., Ura S., Watanabe T., Wada T., Ichijo H., Katada T., Nishina H., EMBO J., 25, 3286-3297 (2006).

13) Watanabe H., Suzuki A., Kobayashi M., Takahashi E., Itamoto M., Lubahn D.B., Handa H., Iguchi T., J. Mol. Endocrinol., 30, 347-358 (2003).

14) Boudjelal M., Taneja R., Matsubara S., Bouillet P., Dolle P., Chambon P., Genes Dev., 11, 2052-2065 (1997).

15) Shimozaki K., Nakashima K., Niwa H., Taga T., Development, 130, 2505-2512 (2003).

16) Singh S., Mishra R., Arango N. A., Deng J. M., Behringer R. R., Saunders G. F., Proc. Natl. Acad. Sci. U.S.A., 99, 6812-6815 (2002).

17) Duncan M. K., Kozmik Z., Cveklova K., Piatigorsky J., Cvek1 A., J. Cell Sci., 113, 3173-3185 (2000).

18) Kralova J., Czerny T., Spanielova H., Ratajova V., Kozmik Z., Gene, 286, 271-282 (2002).

19) Maekawa M., Takashima N., Arai Y., Nomura T., Inokuchi K., Yuasa S., Osumi N., Genes Cells, 10, 1001-1014 (2005). 